\title{
The internet is not limitless
}

\author{
Niamh Kavanagh \\ Department of Physics, UCC
}

"I travel light. But not at the same speed." (Jarod Kintz)

Consider this: you're moving to a new country and you have to fit everything into a single suitcase. Your airline is being very stringent in this situation and you don't want to give them the satisfaction of paying more fees! So, what can you do? You could spend weeks trying to fit it all in, trying different ways to organise everything, but sometimes there's just too much stuff. Well, why not try a completely new, lighter, bigger suitcase? In my research, I try to think the same way about optical fibres.

\section{What's this all about?}

The basis of my $\mathrm{PhD}$ is the development of high-capacity optical communications systems based on new types of optical fibres. In optical communications, digital information is transmitted via optical fibres. Similar to our nervous system, which sends information to different parts of our body via electrical pulses, optical fibres transmit information throughout the world using pulses of light. These fibres are threaded across our whole planet; beneath our feet and under our oceans; connecting cities, countries and continents.

Optical fibres are thin glass fibres, only the thickness of a human hair. If you shine light into the fibre it bounces off the walls and travels along, similar to a ping pong ball down a pipe, as you can see Figure 1.

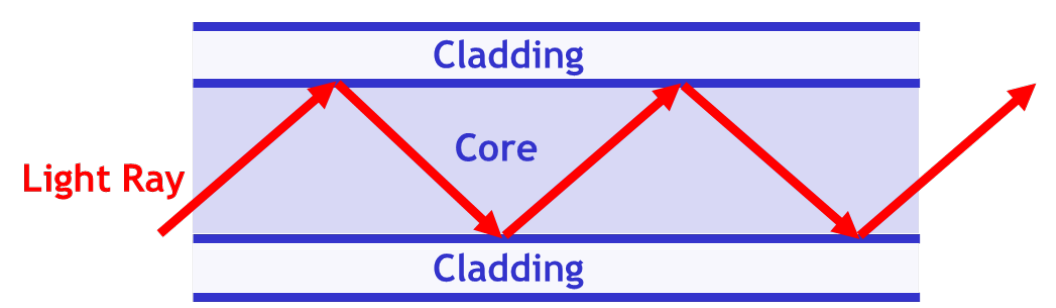

Figure 1: Light travelling in an optical fibre. The fibre is made of glass materials of different densities.The cladding surrounds the core, which is made of denser glass.Source: Niamh Kavanagh

The light signal, provided by a laser, can be encoded with digital information using modulation. Modulation is the addition of information to a light wave by varying properties of the waveform. A simple example of modulation is shown in Figure 2: turning the laser light on to represent binary 1 and off to represent binary 0, utilising Boolean logic similar to Morse code. Digital information has now been translated into pulses of light which 


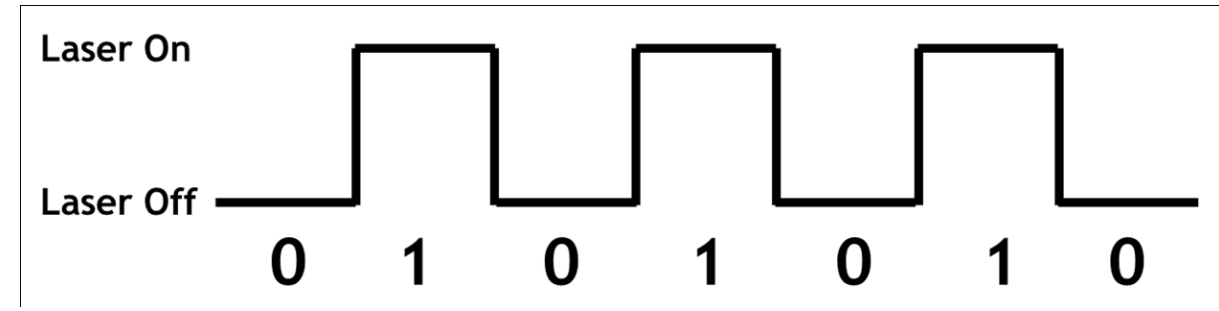

Figure 2: Modulation. Digital information consists of patterns of bits (1's and 0's).These bits can be represented by pulses of light.Source: Niamh Kavanagh.

travel down the fibre at very fast speeds. We can also send several data signals down a single optical fibre at the same time by using light of different colours (i.e. different wavelengths) to carry different data. In this way, optical fibres can carry much more information than copper cables of similar diameter. For example, a network using modern copper lines can carry 3000 telephone calls all at once, while a similar system using fibre optics can carry over 31000 !

\section{Sounds great, what's the problem?}

In today's world, we need to communicate more information than ever before. The internet is not limitless; internet traffic is increasing year-on-year and there is only so much that our current communications systems can handle. Many people now own a mobile phone, tablet, laptop and TV that can all connect to the internet and stream high-quality videos. American multinational technology company, Cisco, predicts that about 50 billion devices will be connected by 2020. Others think the figure could actually be as high as 75 billion - that's 10 times the UN estimate for world population! The "internet of things" is ever-increasing; according to reports, there are 200 unique consumer devices that could be connected to the internet that have not yet done so. Soon, everything from your car to your kettle will send information back and forth over the internet to make sure you have a cup of tea waiting for you when you get home from work! All this information will travel over optical fibres at some point in its transmission.

This increasing use of mobile networks and remote devices is creating a strain on current communication networks and could lead to a global "capacity crunch" in the not-so-distant future. When we talk about capacity we're actually talking about speed, or the amount of data you can transfer in a given time. Less capacity means slower speeds. So, this capacity crunch, if allowed to happen, could seriously impact the internet's future growth meaning slower internet, Skype calls losing connection and more time spent sitting at your laptop watching that little buffering symbol whirl round while you wait for your video to load.

There are several ways to try to combat this capacity crunch. One is to try advanced modulation methods; so instead of just varying the intensity of the light by turning it on and off, you can manipulate other aspects of the light such as its frequency or phase. However, these methods are getting more and more computationally demanding. 


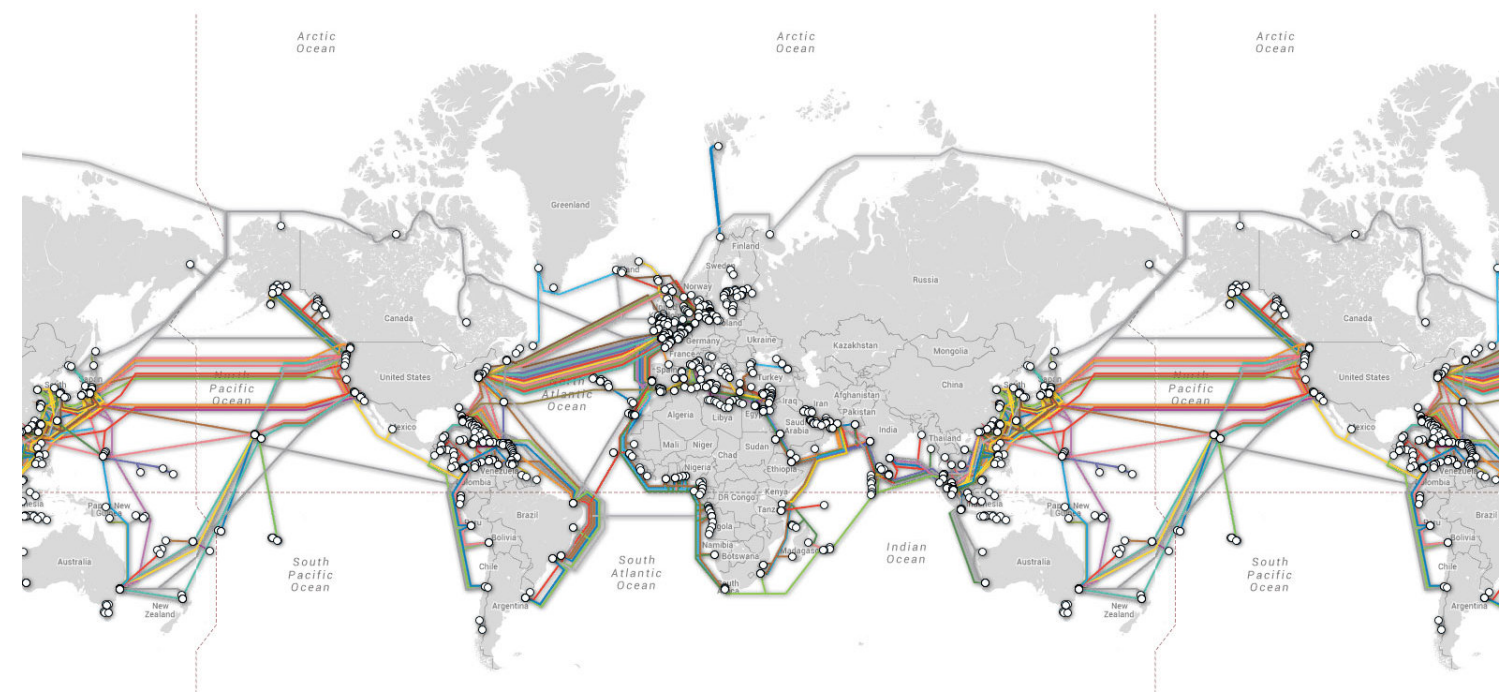

Figure 3: Submarine cable map. This map shows the optical fibre connections under our oceans and seas. These cables form the backbone of our global communications network.Source: Used with permission from TeleGeography:

A second technique is to maximise the use of available bandwidth. Bandwidth refers to the amount of light signals that you can send through the fibre simultaneously (with sufficiently low loss). More bandwidth means you can send more signals and therefore more information. Think of it like increasing the number of traffic lanes on a motorway; more lanes means more traffic can flow. However, we are reaching the maximum bandwidth capability of solid-core fibres.

For long-distance communications, such as transatlantic connections and those shown in Figure 3, the issue of losses becomes very important. As the light signal travels down the fibre it will always incur losses. By gradually leaking out through the fibre walls with each reflection, the signal will fade over distance. To overcome the losses you could increase the power of the signal. However, for high powers, the light signal will begin to interact with the glass of the fibre in unpredictable ways and this can corrupt the information being transmitted.

When the power of the signal is reduced as it travels along, the probability of errors is increased; some 1's will be mistaken for 0's and vice versa. This effect is similar to listening to a radio; at low volume it's difficult to distinguish individual words, so, in order to hear it better you turn up the volume, that is, you increase the power of the sound signal. The same is true for a light signal travelling in a fibre. After a certain distance the signal will need to be amplified in order to provide it with sufficient power to reach its destination and remain clearly distinguishable. The less we have to amplify the signal, the better.

Therefore it is desirable to make sure the losses in the fibres are as low as possible. Solidcore fibres have the lowest loss in the wavelength region of 1.5 micro-meters and, for decades, this wavelength has been the main focus of the optical communications industry. 


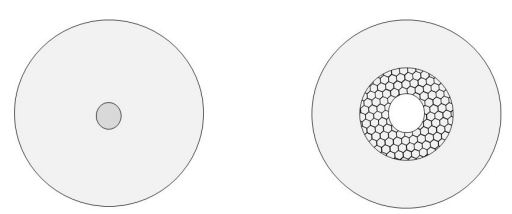

Figure 4: Cross section of solid-core fibre (left): a core of glass is surrounded by a cladding of less dense glass - the light travels in the glass core by bouncing off the core walls; Cross section of hollow-core fibre (right): a core of air is surrounded by a honeycomb structure of glass - the light travels in air by reflecting off the walls in the structure surrounding the core. Source: Niamh Kavanagh.

The current record for lowest loss is around $0.2 \mathrm{~dB} / \mathrm{km}$. For every kilometre the light travels, it loses $0.2 \mathrm{~dB}$ of power. As an example, this means that after $50 \mathrm{~km}, 10 \%$ of the power remains.

We have pushed conventional optical fibres to the limit. We've tried several ways to try and pack it all in (advanced modulation, increasing bandwidth, higher powers), but these methods are becoming more and more complex. New solutions are needed to meet future demands.

\section{So, what's my solution? A new suitcase!}

Instead of trying different ways to pack the information in, let's try a different way of carrying the information! Hollow-Core Photonic Bandgap Fibres (HC-PBGF) may be our answer. These fibres are structured similarly to a drinking straw. A glass tube surrounds a core of air, which means that these fibres guide light through air, rather than glass as before. For example, the difference between solid-core glass fibres and these new hollowcore ones is like running in water versus running outside on a track; the light is no longer impeded by travelling through the glass. The difference in the structures of the two types of fibre is illustrated in Figure 4 (left and right).

Hollow-core fibres have several promising advantages over solid-core fibres. They are predicted to achieve losses as low as $0.1 \mathrm{~dB} / \mathrm{km}$, which means that $10 \%$ of the power remains after $100 \mathrm{~km}$, i.e., double the previous distance of $50 \mathrm{~km}$. This means less amplifiers will be needed for the same distance. Even better, it is thought that these hollow-core fibres are capable of increasing capacity by 10 to 100 times more than that of solid-core fibres! These fibres have three times more bandwidth and since the light signal now interacts with less material as it travels along, we can launch signals into the fibre that are 1000 times more powerful than before. They can carry much more information, farther and faster than we've ever seen.

But what does that mean to us, the users? The total capacity refers to the number of bits (or 1's and 0's) transmitted per second. Let's assume that the total capacity of solid-core fibre is about 10 terabits (that's 1000000000000 bits) per second and that this supports a hundred thousand users (which is roughly the population of Cork city). If hollow-core 
fibres can provide a ten-fold increase in capacity this would mean that one million people, that is the whole of Munster, could then be supported by this communications system!

However, as always, there is a catch. The minimum loss of these new hollow-core fibres is at a longer wavelength of 2 micro-meters (rather than 1.5 micro-meters for solid-core fibres) and this means we need to develop components operable in this new wavelength region.

Now, when talking about components, you can think of an optical communications system like a tin can telephone. You have a transmitter, a receiver and a transmission medium. When my brother and I were little we used to have one of these between our bedrooms. We tried out different tin cans and found the best string, which turned out to be the twine that my Dad used to hold up his tomato plants in the garden. He wasn't too happy when we "borrowed it" for our telephone! But my point is, everything was optimised for our little telephone system. The same is true for optical communications, everything is optimised for solid-core fibres. However, as researchers, we have to ask ourselves, is this really the best system?

\section{What am I doing to help?}

The move from solid-core to hollow-core fibres is, unfortunately, not as simple as switching suitcases. Hence, the goal of my $\mathrm{PhD}$ research is to optimise this new communication system operating at 2 micro-meters, from transmitter to receiver. This includes new lasers, amplifiers, modulators, detectors, filters and much more. The aim of my $\mathrm{PhD}$ is to show that optical communications at 2 micro-meters is possible, with the same performance as current 1.5 micro-meter systems. This is important because, in this way, the expensive electronics associated with optical communications system can remain the same. By upgrading only the optical-side of the system, we can enable much greater bandwidth, lower losses and higher powers, thus significantly increasing the overall capacity by 10 to 100 times.

\section{Conclusion}

So, think back to that suitcase of yours. It has to carry a rather funny collection of travel items - emails, HD videos, Skype calls, and all manner of social media posts. With the growing global demand for faster and larger data transfer, a "capacity crunch" is looming large. New, radical ways of thinking are needed to meet future demands. To me, hollowcore fibres look like a promising possibility. These fibres offer significant improvements over standard solid-core fibres and could increase the overall capacity by up to 100 times! If we can show that optical communications at 2 micro-meters is a viable alternative to 1.5 micro-meter systems, while maintaining the same electronic setup, I think we'll have found our new bigger, lighter and better suitcase for next-generation optical communications! 
I wish to express my sincere thanks to my supervisor Dr. Fatima Gunning for her support, guidance and motivation. I am also grateful to my family and friends (shout-out to Mike, Brian, Shane and Jessie) for their proof-reading, reassurance and constant encouragement. I would like to acknowledge the Irish Research Council (IRC) as the funding body for my research, as well as the EU FP7 IP project MODEGAP (258033), SFI projects 12/RC/2276 (IPIC) and 13/CDA/2103. Thanks also to the staff of the Department of Physics (UCC) and the Photonics Systems Group (Tyndall National Institute). 